\title{
openheart CT imaging prior to transcatheter aortic valve implantation in the UK
}

\author{
Iwan Harries (D) , ${ }^{1}$ Jonathan R Weir-McCall (D) , ${ }^{2}$ Michelle C Williams, ${ }^{3}$ \\ James Shambrook, ${ }^{4}$ Giles Roditi, ${ }^{5}$ Russel Bull, ${ }^{6}$ Gareth J Morgan-Hughes, ${ }^{7}$ \\ Edward D Nicol, ${ }^{8}$ Alastair J Moss (1) ${ }^{9}$
}

\begin{abstract}
- Additional material is published online only. To view please visit the journal online (http://dx.doi.org/10.1136/ openhrt-2019-001233).
\end{abstract}

To cite: Harries I, Weir-McCall JR, Williams MC, et al. CT imaging prior to transcatheter aortic valve implantation in the UK. Open Heart 2020;7:e001233.

doi:10.1136/

openhrt-2019-001233

Received 29 December 2019 Revised 25 January 2020 Accepted 17 February 2020

Check for updates

(c) Author(s) (or their employer(s)) 2020. Re-use permitted under CC BY-NC. No commercial re-use. See rights and permissions. Published by BMJ.

${ }^{1}$ Cardiology, Bristol Heart Institute, Bristol, UK

${ }^{2}$ Radiology, University of Dundee School of Medicine, Dundee, UK ${ }^{3}$ Centre for Cardiovascular Sciences, University of Edinburgh, Edinburgh, UK ${ }^{4}$ Radiology, University Hospital Southampton NHS Foundation Trust, Southampton, UK ${ }^{5}$ Radiology, Glasgow Royal Infirmary, Glasgow, UK ${ }^{6}$ Radiology, Royal Bournemouth Hospital, Bournemouth, UK ${ }^{7}$ Cardiology, Plymouth Hospitals NHS Trust, Plymouth, UK

${ }^{8}$ Cardiology, Royal Brompton and Harefield NHS Foundation Trust, London, UK

${ }^{9}$ University of Edinburgh Centre for Cardiovascular Sciences, Edinburgh, UK

Correspondence to Dr Iwan Harries; iwanharries@ hotmail.com

\section{ABSTRACT}

Objective This cross-sectional observational study sought to describe variations in $\mathrm{CT}$ in the context of transcatheter aortic valve implantation (CT-TAVI) as currently performed in the UK.

Methods 408 members of the British Society of Cardiovascular Imaging were invited to complete a 27item online CT-TAVI survey.

Results 47 responses (12\% response rate) were received from 40 cardiac centres, $23(58 \%)$ of which performed TAVI on-site (TAVI centres). Only six respondents (13\%) performed high-volume activity (>200 scans per year) compared with 13 (28\%) performing moderate (100-200 scans per year) and 27 (59\%) performing low (0-99 scans per year) volume activity. Acquisition protocols varied (41\% retrospective, $12 \%$ prospective with wide padding, $47 \%$ prospective with narrow padding), as did the phase of reporting ( $45 \%$ systolic, $37 \%$ diastolic, $11 \%$ both, $6 \%$ unreported). Median dose length product was 675 mGy. cm (IQR 477-954 mGy.cm). Compared with non-TAVI centres, TAVI centres were more likely to report minimum iliofemoral luminal diameter $(n=25,96 \%$ vs $n=7,58 \%$, $\mathrm{p}=0.003$ ) and optimal tube angulation for intervention ( $n=12,46 \%$ vs $n=1,8 \%, p=0.02$ ).

Conclusions This national survey formally describes current CT-TAVI practice in the UK. High-volume activity was only present at one in seven cardiac CT centres. There is wide variation in scan acquisition, scan reporting and radiation dose exposure in cardiac CT centres.

\section{INTRODUCTION}

Percutaneous transcatheter aortic valve implantation (TAVI) for severe aortic valve disease has become widespread over the past decade. The improved safety profile of TAVI compared with cardiothoracic surgery in lower risk cohorts is likely to result in the further expansion of TAVI in the coming years. ${ }^{1}$ Historically, cardiothoracic surgery has offered direct visualisation of the cardiac anatomy to plan and implement appropriate valve replacement, but the advent of TAVI has necessitated a preprocedural road map of the cardiac anatomy to facilitate the safe deployment of appropriately sized aortic valve prostheses. $^{2}$ The isotropic resolution of cardiac CT has made it a critical investigation in

\section{Key questions}

What is already known about this subject?

- CT is pivotal in guiding patient selection, valve sizing and determining procedural risk assessment prior to transcatheter aortic valve implantation (TAVI). It is recognised that TAVI practice varies according to institutional infrastructure and physician training and preference but the extent to which practice varies is not known.

What does this study add?

- This study provides an overview of current CT-TAVI acquisition, reporting and dissemination of findings in the UK and serves as a point of reference for individuals and organisations seeking to evaluate their own methods of performing CT-TAVI.

How might this impact on clinical practice?

- Greater insight into current national trends in CTTAVI practice may inform changes to individual institutional protocols and facilitate 'hub and spoke' communication between TAVI and non-TAVI centres.

assessing valve anatomy when deciding which patients may be suitable for TAVI. ${ }^{3}$

While standards of practice exist for cardiac CT in the assessment of coronary artery disease,${ }^{4}$ there is a paucity of data regarding the optimal image acquisition protocols and standardised reporting criteria for CT in TAVI (CT-TAVI) scans. Recently, the Society of Cardiovascular Computed Tomography has published an expert consensus document highlighting the need for standardisation of nomenclature and measurements using CT-TAVI. ${ }^{5}$ However, whether these international recommendations reflect the current practice of CT-TAVI acquisition and reporting in the UK is unknown. Additionally, while diagnostic dose reference levels are available for most CT procedures, the acceptable limits of ionising radiation for CT-TAVI are yet to be established. ${ }^{6}$

This study aimed to survey members of a national cardiovascular imaging society who 
perform CT-TAVI to assess the variability in scan acquisition, reporting and dissemination of findings.

\section{METHODS}

\section{Survey population}

All 408 members of the British Society of Cardiovascular Imaging (BSCI) were invited to complete a 27-item online survey (online supplementary file 1) (www.surveymonkey. com) in April 2018. The BSCI is composed of members of the cardiovascular imaging community, which serves as a Specialist Advisory Group on matters related to cardiovascular imaging to the British Cardiovascular Society and Royal College of Radiologists, UK. While standards of practice for the training and reporting of CT coronary angiography (CTCA) have been produced by the BSCI in line with recommendations from the Francis report, ${ }^{7}$ no standards of practice exist for the training and reporting of CT-TAVI scans. Survey questions were constructed to assess the level of activity, and variability in CT-TAVI practice, across centres in the UK currently delivering this service for the National Health Service (NHS) England, NHS Wales, NHS Scotland, and Health and Social Care in Northern Ireland. Patients and the public were not involved in the design of the survey.

\section{Items surveyed}

All participants were surveyed individually to prevent collaboration bias between institutions. All responses were included in the analysis. Parameters recorded included the type of institution (centres performing TAVI (TAVI centre) vs those without a TAVI (non-TAVI centre) programme), make of CT scanner, total number of CT-TAVI scans performed annually, scan acquisition protocol, use of medication (beta-blockers) during scan acquisition, contrast volume and radiation doses of the last five scans performed, structured reporting metrics of the aortic valve annulus and vascular access, and access to and distribution of findings to the TAVI multidisciplinary team.

\section{Statistical analysis}

Continuous variables are reported as mean $\pm \mathrm{SD}$ or median and IQR, for normally and non-normally distributed variables, respectively. The survey questions were built using skip logic to save respondents time and ensure a high response rate. The skip or 'conditional branching' pattern varies depending on the respondent's answers and this results in incomplete data sets for some survey questions. Consequently, categorical variables are presented as counts and percentages of the total number of complete responses for each question. The survey was designed as a descriptive exploration of current practices and thus not designed nor powered to detect specific differences. Where differences in practice were apparent, continuous variables were assessed with Student's t-test and categorical variables were assessed with Fisher's exact test in cases where $\chi^{2}$ test was not valid. A $p$ value $<0.05$ was taken to indicate statistical significance. The analysis was carried out in SPSS (IBM SPSS Statistics for Macintosh, V.25.0. IBM).

\section{RESULTS}

\section{CT-TAVI annualised activity in TAVI and non-TAVI centres}

Forty-seven responses (12\% response rate) from 40 cardiac centres in the UK were received (figure 1). Fifty-three per cent of respondents $(n=25 / 47)$ were radiologists and $47 \%$ $(n=22 / 47)$ were cardiologists with a subspecialty interest in cardiac CT. Most respondents worked in centres which included a TAVI programme on-site $(\mathrm{n}=30 / 47,63.8 \%)$ (figure 1). Annualised volume of CT-TAVI activity for acquisition and reporting varied considerably $(\mathrm{n}=1$, no data provided), $59 \%$ of respondents $(n=27 / 46)$ reported a low volume of activity ( $<100$ CT-TAVI scans per annum), compared with $28 \%(\mathrm{n}=13 / 46)$ performing a moderate level of activity (100-200 scans per annum) and 13\% $(n=6 / 46)$ performing a high level of scan activity $(>200$ scans per annum) (figure 1). Respondents working in TAVI centres performed more CT-TAVI activities per annum compared with those in non-TAVI centres $(\mathrm{p}<0.001)$.

\section{Scan acquisition and radiation dose in TAVI and non-TAVI centres}

Overall, retrospective ECG-gated acquisitions were performed by $41 \% \quad(n=14 / 34)$ of respondents, compared with $47 \% \quad(\mathrm{n}=16 / 34)$ performing prospective ECGtriggered acquisitions with narrow padding, and 12\% $(n=4 / 34)$ performing prospective ECG-triggered acquisitions with wide padding (figure 2). There was no difference between TAVI and non-TAVI centres regarding acquisition protocol $(\mathrm{p}=0.72)$. Median contrast volumes and dose length product (DLP) of the five most recently performed CT-TAVI scans did not vary between TAVI and non-TAVI centres (table 1). The median CT-TAVI DLP across all participating centres was $675 \mathrm{mGy} . \mathrm{cm}$ (IQR 477-954 mGy.cm). Using wide padding (30\%-80\% R-R interval) more than doubles the CT-TAVI DLP from 423 to $921 \mathrm{mGy} . \mathrm{cm}$ for prospectively triggered CT-TAVI, whereas retrospective ECG gating yielded a median DLP of $882 \mathrm{mGy.cm}$ (table 1). Beta-blockade was administered by $15 \%(n=7 / 47)$ of respondents. Furthermore, cardiologists were significantly more likely to administer betablockers compared with radiologists $(n=6 / 25,24 \%$ vs $\mathrm{n}=1 / 22,5 \%, \mathrm{p}=0.01$ ).

\section{CT-TAVI reporting in TAVI and non-TAVI centres}

Thirty-five centres provided parameters on reporting. Two centres acquired scans but did not analyse preprocedural measurements $(6 \%)$. Forty-five per cent $(n=16 / 35)$ of centres reported systolic-only aortic annulus measurements, $37 \%(\mathrm{n}=13 / 35)$ reported diastolic-only aortic annulus measurements and $11 \% \quad(n=4 / 35)$ reported both systolic and diastolic aortic annulus measurements. Five centres did not report the phase of reconstruction. With respect to the detailed assessment of aortic root measurements, there were no standard metrics which 


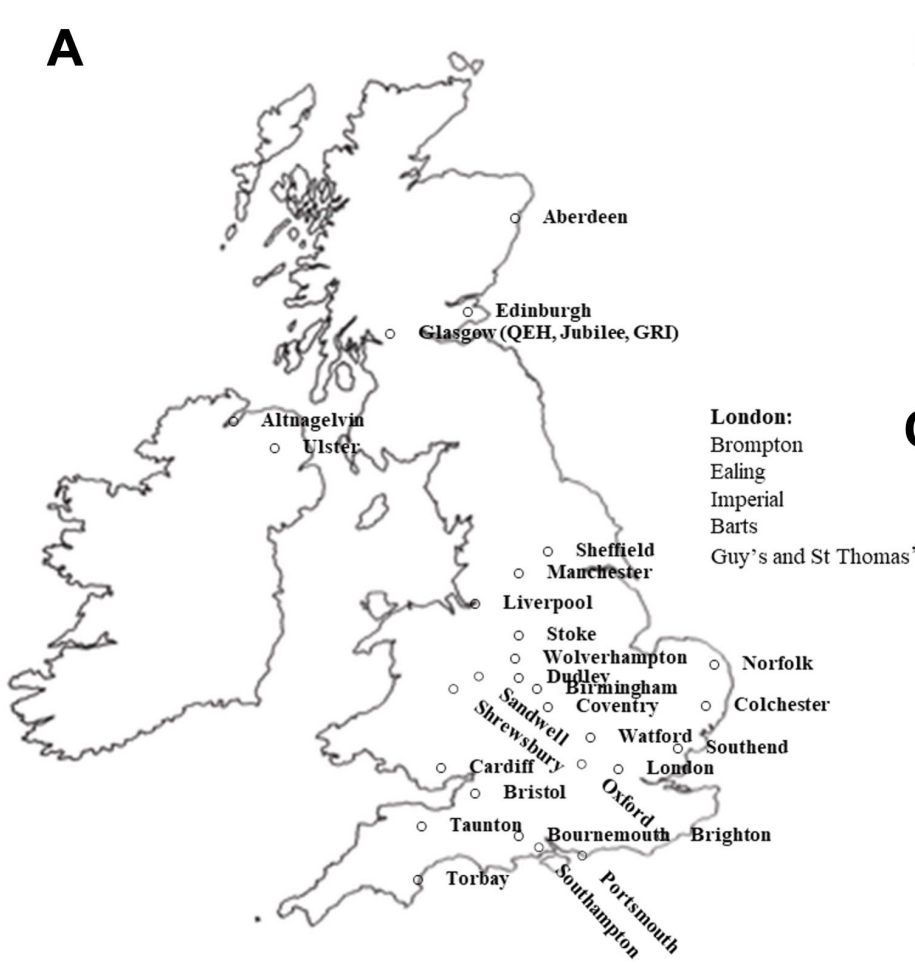

B
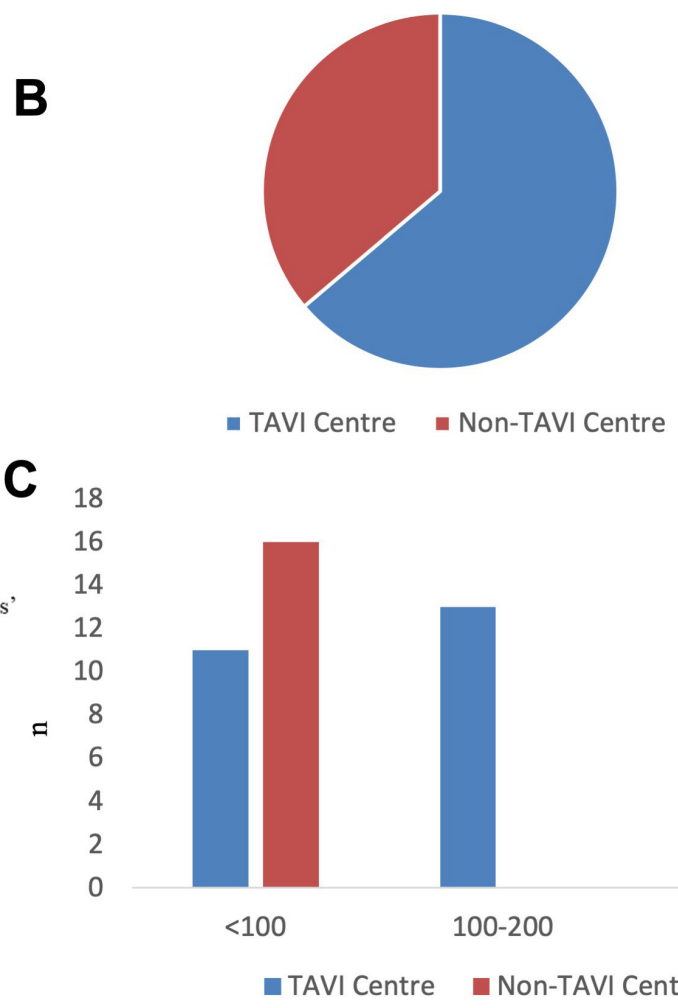

- TAVI Centre $=$ Non-TAVI Centre

Figure 1 Geographical distribution of survey respondents. Cardiac CT centres from England, Scotland, Wales and Northern Ireland provided survey data (A). Sixty-five per cent of respondents worked in centres that provided a transcatheter aortic valve implantation (TAVI) programme (B). Annualised activity varied across centres with non-TAVI centres performing $<100$ scans per annum (C).

were reported by all centres (table 2). Interestingly, coronary artery luminal stenosis severity was included in all CT-TAVI reports at $24 \%(\mathrm{n}=9 / 38)$ of centres and sometimes reported in 50\% ( $\mathrm{n}=19 / 38)$ of centres. Compared with non-TAVI centres, TAVI centres were more likely to report minimum iliofemoral luminal diameter $(n=25 / 26$, $96 \%$ vs $\mathrm{n}=7 / 12,58 \%, \mathrm{p}=0.003)$ and optimal tube angulation for intervention $(\mathrm{n}=12 / 26,46 \%$ vs $\mathrm{n}=1 / 12,8 \%$, $\mathrm{p}=0.02$ ) (table 2). There were no significant differences in reporting parameters when compared by specialty.

\section{'Heart Team' dissemination of findings}

A 'Heart Team' discussion involving a cardiac imaging specialist is more frequent in TAVI centres compared with non-TAVI centres $(96 \%, \mathrm{n}=24 / 25$ vs $33 \%, \mathrm{n}=4 / 12$, $\mathrm{p}<0.001$ ) (figure 3). However, to facilitate interpretation of CT-TAVI findings, a cardiac imaging specialist is 'always present' at $57 \%(\mathrm{n}=16 / 28)$ of centres overall $(54 \%$, $\mathrm{n}=13 / 24$ TAVI centres vs $75 \%, \mathrm{n}=3 / 4$ non-TAVI centres), and 'most of the time' at a further $21 \%(n=6 / 28)$ of
A

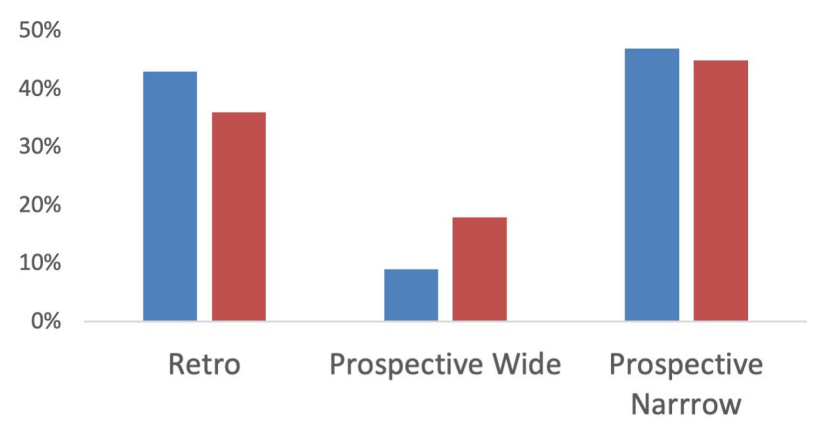

B

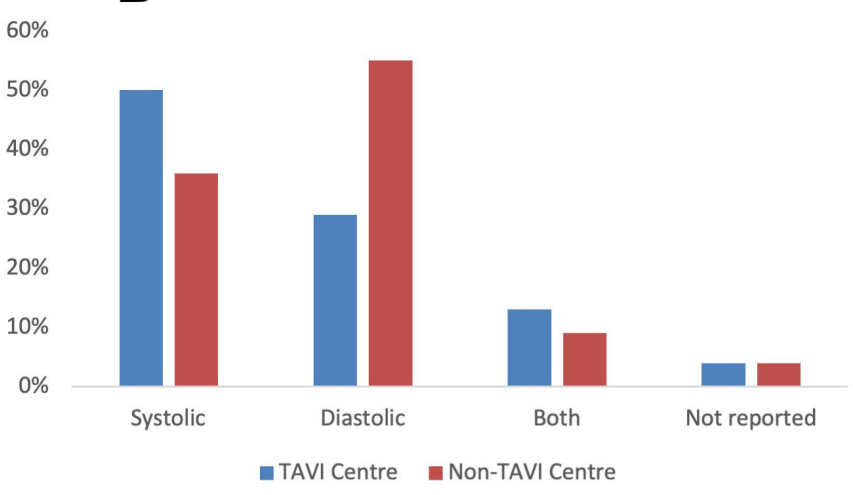

TAVI Centre non-TAVI Centre

Figure 2 CT-TAVI scan acquisition protocols. Image acquisition protocols according to centre type (A). Phase of measurement reporting according to centre type (B). TAVI, transcatheter aortic valve implantation. 
Table 1 Estimated contrast volumes and dose length product (DLP) for the last five CT-TAVI scans performed at each centre and radiation doses according to scan protocol

\begin{tabular}{lllll}
\hline & Overall & TAVI centres $(\mathbf{n}=22)$ & Non-TAVI centres $(\mathbf{n}=\mathbf{9})$ & P value \\
\hline Contrast volume $(\mathrm{mL})$ & $100(80-130)$ & $95(74-120)$ & $110(88-160)$ & 0.06 \\
& Min 50, max 170 & Min 60, max 150 & Min 50, max 170 & \\
DLP (mGy.cm) & $675(477-954)$ & $550(423-939)$ & $700(538-1075)$ & 0.66 \\
& Min 139, max 2000 & Min 145, max 2000 & Min 139, max 1750 & \\
\hline
\end{tabular}

CT-TAVI scan protocol

\begin{tabular}{lll}
\hline $\begin{array}{l}\text { Retrospective-whole cardiac } \\
\text { cycle }(\mathbf{n}=12)\end{array}$ & $\begin{array}{l}\text { Prospective ECG-gated } \\
\text { narrow padding }(\mathbf{n}=\mathbf{1 2})\end{array}$ & $\begin{array}{l}\text { Prospective ECG-gated wide } \\
\text { padding }(\mathbf{n}=4)\end{array}$ \\
\hline $882(524-1688)^{\star}$ & $423(230-631)$ & $921(850-1138)^{\star *}$
\end{tabular}

$\operatorname{DLP}(m G y . c m)$

$882(524-1688)^{*}$

$423(230-631)$

$921(850-1138)^{\star \star}$

Values are median and IQR.

${ }^{*} \mathrm{P}=0.003,{ }^{* *} \mathrm{p}=0.002$, both versus prospective ECG-gated narrow padding.

TAVI, transcatheter aortic valve implantation.

Table 2 Measurements and structures typically included in the CT-TAVI report at each centre

\begin{tabular}{|c|c|c|c|c|}
\hline & Overall $(n=38)$ & $\begin{array}{l}\text { TAVI centres } \\
(\mathrm{n}=26)\end{array}$ & $\begin{array}{l}\text { Non-TAVI } \\
\text { centres }(n=12)\end{array}$ & $P$ value \\
\hline Aortic annulus circumference & 21 (55\%) & $16(62 \%)$ & $5(42 \%)$ & 0.25 \\
\hline Aortic annulus area & $24(63 \%)$ & $17(65 \%)$ & $7(58 \%)$ & 0.675 \\
\hline Aortic annulus diameter (perimeter derived) & $23(61 \%)$ & $17(65 \%)$ & $6(50 \%)$ & 0.367 \\
\hline Aortic annulus diameter (area derived) & $19(50 \%)$ & $13(50 \%)$ & $6(50 \%)$ & 1.0 \\
\hline $\begin{array}{l}\text { Minimum short-axis measurement } \\
\text { Sinus of Valsalva diameter (cusp-cusp) }\end{array}$ & $20(53 \%)$ & $14(54 \%)$ & $6(50 \%)$ & 0.82 \\
\hline $\begin{array}{l}\text { Minimum short-axis measurement } \\
\text { Sinus of Valsalva diameter (cusp-commissure) }\end{array}$ & $20(53 \%)$ & $13(50 \%)$ & $7(58 \%)$ & 0.63 \\
\hline Extent and distribution of aortic root calcification & $31(82 \%)$ & $22(85 \%)$ & $9(75 \%)$ & 0.48 \\
\hline Number of valve cusps (eg, bicuspid, tricuspid) & $32(84 \%)$ & $22(85 \%)$ & $10(83 \%)$ & 0.92 \\
\hline Distance (height) from aortic annulus to LMS ostium & $30(79 \%)$ & $21(81 \%)$ & $9(75 \%)$ & 0.69 \\
\hline Distance (height) from aortic annulus to RCA ostium & $30(79 \%)$ & $21(81 \%)$ & $9(75 \%)$ & 0.69 \\
\hline Distance (height) from aortic annulus to sinotubular junction (left cusp) & $11(29 \%)$ & $6(23 \%)$ & $5(42 \%)$ & 0.24 \\
\hline Distance (height) from aortic annulus to sinotubular junction (right cusp) & $9(24 \%)$ & $5(19 \%)$ & $4(33 \%)$ & 0.34 \\
\hline $\begin{array}{l}\text { Distance (height) from aortic annulus to sinotubular junction (non- } \\
\text { coronary cusp) }\end{array}$ & $7(18 \%)$ & $4(15 \%)$ & $3(25 \%)$ & 0.48 \\
\hline Presence of LVH & $23(61 \%)$ & $18(69 \%)$ & $5(42 \%)$ & 0.17 \\
\hline Ascending aorta diameter & $31(82 \%)$ & $21(81 \%)$ & $10(83 \%)$ & 0.85 \\
\hline $\begin{array}{l}\text { Descending and abdominal aorta (tortuosity, intraluminal obstruction, } \\
\text { calcification) }\end{array}$ & $31(82 \%)$ & $23(88 \%)$ & $8(67 \%)$ & 0.11 \\
\hline Subclavian and brachiocephalic artery diameter & $18(47 \%)$ & $16(62 \%)$ & $2(17 \%)$ & 0.01 \\
\hline Minimum iliofemoral artery luminal diameter & $32(84 \%)$ & $25(96 \%)$ & $7(58 \%)$ & 0.003 \\
\hline Iliofemoral artery patency & $32(84 \%)$ & $24(92 \%)$ & $8(67 \%)$ & 0.04 \\
\hline lliofemoral artery tortuosity & $31(82 \%)$ & $24(92 \%)$ & $7(58 \%)$ & 0.01 \\
\hline $\begin{array}{l}\text { Optimal tube angulation data to inform fluoroscopic projection for device } \\
\text { deployment }\end{array}$ & $13(34 \%)$ & $12(46 \%)$ & $1(8 \%)$ & 0.02 \\
\hline
\end{tabular}

Values are count $(n)$ and percentage (\%).

Bold values indicate statistical significance at a $p$ value $<0.05$

LMS, leftmain stem; LVH, left ventricular hypertrophy; RCA, right coronary artery; TAVI, transcatheter aortic valve implantation. 


\section{A}
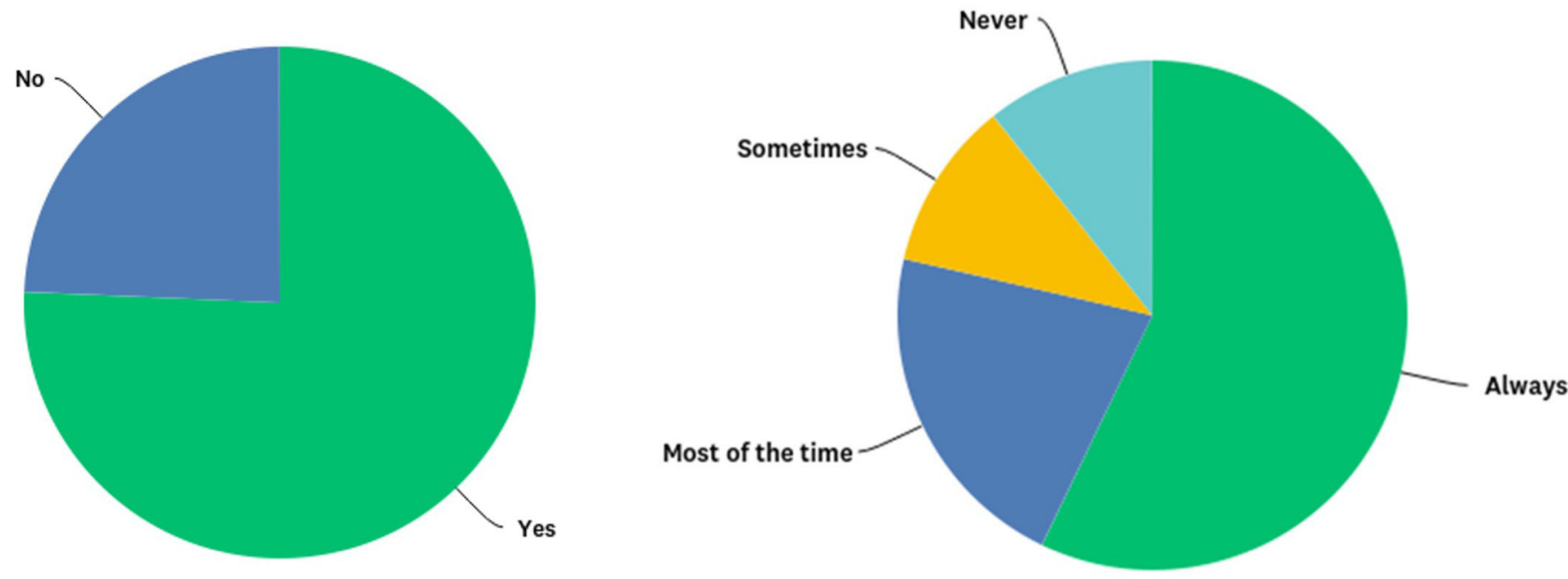

C

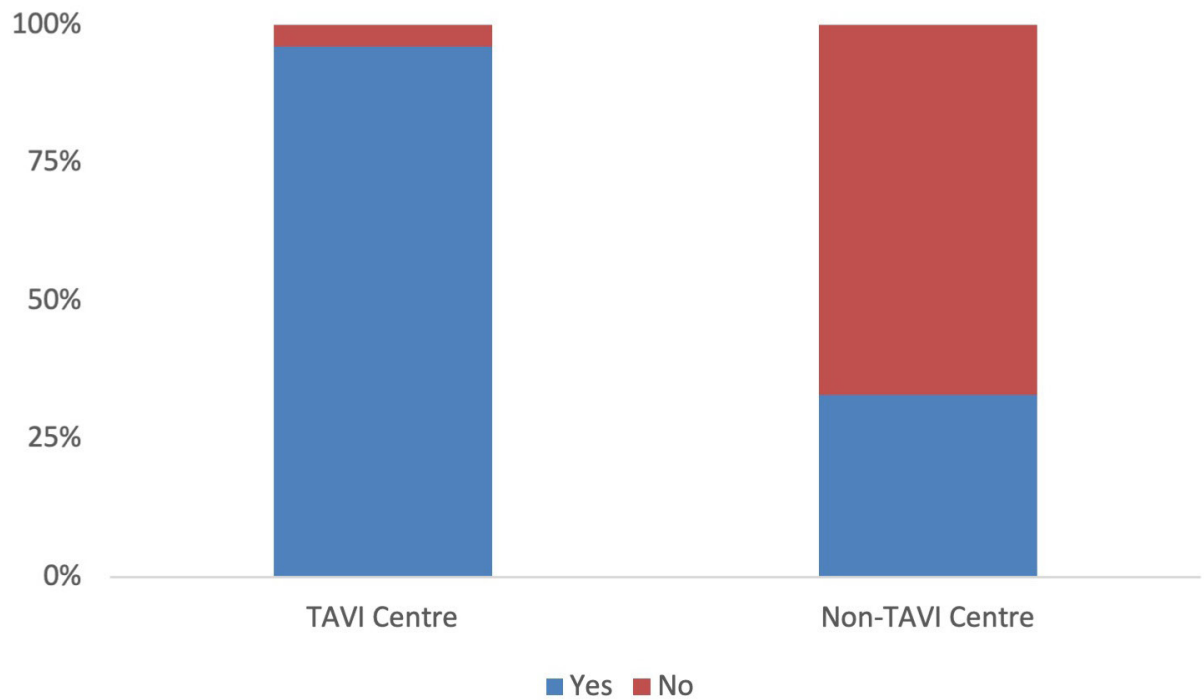

Figure 3 Dissemination of findings to Heart Team. Heart Team multidisciplinary discussions were present at $76 \%$ of centres overall (A). When Heart Team discussions were in place, a cardiac imaging specialist was 'always present' at $57 \%$ of meetings (B). There was a significant difference in the provision of Heart Team meetings at TAVI and non-TAVI centres $(96 \%, n=24 / 25$ vs $33 \%, n=4 / 12, p<0.001)(C)$. TAVI, transcatheter aortic valve implantation.

centres overall $(21 \%, \mathrm{n}=5 / 24$ TAVI centres vs $25 \%, \mathrm{n}=1 / 4$ non-TAVI centres). A cardiac imaging specialist is 'never' present at $11 \%(n=3 / 28)$ of centres $(13 \%, n=3 / 24$ TAVI centres vs $0 \%, \mathrm{n}=0$ non-TAVI centres).

\section{DISCUSSION}

In this study, we found that only one in seven respondents perform a high annualised volume of CT-TAVI . As such, there is wide variation in practice among UK cardiac centres in the acquisition and reporting of CT-TAVI. Median radiation dose exposure was $675 \mathrm{mGy} . \mathrm{cm}$ in UK cardiac centres, however significant reductions in the DLP were obtained by using prospective ECG gating with narrow-padded acquisitions. To ensure optimal reporting standards in all UK cardiac centres, further collaboration between TAVI and non-TAVI centres should be encouraged.

Following the expansion of TAVI over the past decade, the provision of non-invasive cardiac imaging to support structural heart disease programmes can no longer be solely delivered in tertiary cardiac centres. This comes at a time when there has been considerable service expansion in cardiac CT following the implementation of the revised National Institute for Health and Care Excellence Clinical Guideline 95 (update 2016) necessitating that the UK, as a whole, provides over 350000 cardiac CT angiograms per annum. ${ }^{8}$ This compares to the estimated 42340 scans reported by 198 accredited individuals on 304 CTCA-capable scanners recently being performed. ${ }^{9}$ This has resulted in even the highest volume cardiac CT 
centres needing to double their service provision for coronary artery disease assessment, and thus hindering further expansion of cardiac CT in the assessment of structural heart disease in tertiary cardiac centres. ${ }^{9}$ However, an increasing number of new centres which offer cardiac CT has meant that experienced imaging cardiologists and radiologists can also provide lower numbers of CT-TAVI in house remote from TAVI centres, thereby performing CT-TAVI at a location which may be more convenient for the patient. Whether a 'hub and spoke' model that acquires CT imaging at the local centre and offers shared reporting with structural imaging specialists at TAVI centres will improve CT interpretation in nonTAVI centres is unknown. However, this model has been successfully employed in other collaborative networks using CT imaging. ${ }^{10}$

With respect to radiation dose, the 'as low as reasonably achievable' principle is a central tenant of legislation stating that ionising radiation should be optimised to use the minimum amount of radiation to attain adequate diagnostic information. ${ }^{11}$ For coronary CT angiography, the UK has the lowest international diagnostic reference levels set by Ionising Radiation (Medical Exposure) Regulations at $380 \mathrm{mGy} . \mathrm{cm}$ for retrospective acquisitions with ECG gating and $170 \mathrm{mGy} . \mathrm{cm}$ for prospective acquisitions with no padding. ${ }^{12}$ Focusing attention on the optimal scan acquisition in the field of coronary CT angiography has resulted in many centres switching from retrospective to prospective acquisitions and reducing tube current potential, thereby reducing the median radiation exposure by $78 \%{ }^{13}{ }^{14}$ In this study, the wide variation in scanning techniques for acquiring CT-TAVI data sets would suggest that there is scope to lower radiation dose exposure through improved standardisation of practice across UK cardiac CT centres. Indeed, this would fall under the remit of the 'Getting it right first time' model that aims to reduce unwarranted variation in UK clinical practice. Of note, radiation doses reported by the members surveyed varied 14-fold between the lowest and highest reported DLPs (range 139-2000 mGy.cm). Some of this variation may be attributed to the perceived need to analyse the aortic annulus in both ventricular systole and diastole. While there is no consensus on the optimal acquisition technique, international guidelines advocate a preference for systolic scan coverage, as this is when the aortic annulus is most circular and has the largest orifice area for accurate valve sizing. ${ }^{51516}$ However, reverse dynamism of the interventricular septum which is frequently seen in patients with sigmoid hypertrophy may favour dimensions measured in ventricular diastole, thus necessitating retrospective ECG-gated reconstructions throughout the entire cardiac cycle. ${ }^{5}$ Further investigation of the optimal CT-TAVI acquisition for routine clinical practice is warranted.

Decisions regarding TAVI are made in the context of a 'Heart Team' multidisciplinary discussion which draws on expertise from interventional cardiologists, cardiac surgeons and cardiac imaging specialists. ${ }^{17}$ While local institutions may differ in their approach to the composition of the 'Heart Team', it is unsurprising that these are more likely to occur in TAVI centres than non-TAVI centres. However, it is notable that despite the critical importance of CT-TAVI in the preprocedural planning of transcatheter heart valve procedures, there are some TAVI centres where a cardiac imaging specialist is never present to interpret the imaging findings in the clinical context. This may explain why the aortic annular dimension evaluation, a critical step for transcatheter aortic valve sizing, differs in the level of detailed reporting between sites. In order to maximise the information obtained from CT-TAVI scans both in terms of written reporting and dissemination to interventional cardiologists, cardiac imagers should be integrated into the 'Heart Team' discussion. This would help address specific questions in CT-TAVI reporting, such as which parameters constitute a mandatory minimum for a CT-TAVI report. ${ }^{18}$ In this regard, the observation that coronary artery stenosis assessment is performed in up to $50 \%$ of cardiac CT centres raises the question as to whether this should be routinely reported on CT-TAVI scans. ${ }^{19} 20$ The use of CT-TAVI to evaluate the patency of coronary artery bypass grafts and native coronary arteries is feasible, however as periprocedural medication (beta-blocker, glyceryl trinitrate) that is routinely administered for coronary CT angiography is contraindicated in the setting of severe aortic stenosis, optimal images may not always be obtained. ${ }^{20}$ Interestingly, while selective beta-blockade has been used in the context of severe asymptomatic ${ }^{21}$ and symptomatic aortic stenoses, ${ }^{22}$ there is a paucity of evidence supporting the safety of intravenous metoprolol administration during CT-TAVI acquisition. If beta1selective blockade is to be considered on an individual basis, the patient should be counselled on the risks of exacerbating symptoms related to aortic stenosis. Medication use at the time of CT-TAVI will be explored in more detail in future studies.

There are some limitations to this study. Despite attempts to obtain responses from UK cardiac CT centres, some centres that perform CT-TAVI were not captured. Moreover, the survey only elicited responses (response rate only $12 \%$ ) from members of the BSCI, meaning that an unknown but likely significant number of those reporting CT-TAVI, for example, interventional cardiologists, have not been captured. This introduces bias and influences the external validity of the data, while reliance on selfreporting of data may also introduce error. Furthermore, some responses from different individuals originated from the same centre. Because these data were frequently discordant, reflecting intracentre variation, we elected to include all responses from all respondents, which is also a source of bias. The image quality of CT-TAVI scans obtained at UK cardiac CT centres was not analysed in this study, but we presume that each centre obtains diagnostic CT-TAVI images on the majority of occasions. Finally and perhaps most importantly, no data on complications following CT-TAVI (eg, contrast-induced nephropathy) 
were collected. Future studies exploring the relationship between CT-TAVI, periprocedural complications and patient outcomes would be instructive and could inform optimal CT-TAVI scan acquisition protocols.

\section{CONCLUSION}

This national survey formally describes current CT-TAVI practice in the UK. High-volume activity was only present at one in seven cardiac CT centres. There is wide variation in scan acquisition, scan reporting and radiation dose exposure in cardiac CT centres.

Contributors $\mathbb{H}$ helped design the research, collected, analysed and interpreted the data, drafted and revised the manuscript critically for important intellectual content. JWM and MCW analysed the data and revised the manuscript critically for important intellectual content. JS, GR, RB and GJMH revised the manuscript critically for important intellectual content. EN helped design the research, interpreted the data and revised the manuscript critically for important intellectual content. AJM conceived and designed the research, analysed and interpreted the data, redrafted the manuscript and revised it critically for important intellectual content. All authors approved the final version of the manuscript and agree to be accountable for all aspects of the work in ensuring that questions related to the accuracy or integrity of any part of the work have been appropriately investigated and resolved.

Funding This study was supported by a grant from the British Society of Cardiovascular Imaging/British Society of Cardiac Computed Tomography. The British Society of Cardiovascular Imaging/British Society of Cardiac Computed Tomography is a registered charity (1145324).

Competing interests None declared.

Patient consent for publication Not required.

Ethics approval Owing to the study design, ethical approval was not required prior to distribution of the survey.

Provenance and peer review Not commissioned; externally peer reviewed.

Data availability statement Data are deidentified questionnaire responses available from the corresponding author upon reasonable request.

Open access This is an open access article distributed in accordance with the Creative Commons Attribution Non Commercial (CC BY-NC 4.0) license, which permits others to distribute, remix, adapt, build upon this work non-commercially, and license their derivative works on different terms, provided the original work is properly cited, appropriate credit is given, any changes made indicated, and the use is non-commercial. See: http://creativecommons.org/licenses/by-nc/4.0/.

\section{ORCID iDs}

Iwan Harries http://orcid.org/0000-0003-0688-3345

Jonathan R Weir-McCall http://orcid.org/0000-0001-5842-842X

Alastair J Moss http://orcid.org/0000-0003-4123-2070

\section{REFERENCES}

1 Mack MJ, Leon MB, Thourani VH, et al. Transcatheter aortic-valve replacement with a balloon-expandable valve in low-risk patients. $N$ Engl J Med 2019;380:1695-705.

2 Piazza N, de Jaegere P, Schultz C, et al. Anatomy of the aortic valvar complex and its implications for transcatheter implantation of the aortic valve. Circ Cardiovasc Interv 2008;1:74-81.

3 Achenbach S, Delgado V, Hausleiter J, et al. SCCT expert consensus document on computed tomography imaging before transcatheter aortic valve implantation (TAVI)/transcatheter aortic valve replacement (TAVR). J Cardiovasc Comput Tomogr 2012;6:366-80.

4 Harden S. . BSCl / RCR / RCP Standards of practice of computed tomography coronary angiography (CTCA) in adult patients: British Society of Cardiovascular Imaging/British Society of Cardiac CT. Available: http://www.bsci.org.uk

5 Blanke P, Weir-McCall JR, Achenbach S, et al. Computed Tomography Imaging in the Context of Transcatheter Aortic Valve Implantation (TAVI)/Transcatheter Aortic Valve Replacement (TAVR): An Expert Consensus Document of the Society of Cardiovascular Computed Tomography. JACC Cardiovasc Imaging 2019;12:1-24.

6 Williams MC, Stewart C, Weir NW, et al. Using radiation safely in cardiology: what imagers need to know. Heart 2019;105:798-806.

7 The Mid Staffordshire NHS Foundation Trust Public Inquiry. Chaired by Robert Francis QC. Report of the mid Staffordshire NHS Foundation trust public inquiry. London: The Stationary Office, 2013.

8 Moss AJ, Williams MC, Newby DE, et al. The updated NICE guidelines: cardiac CT as the first-line test for coronary artery disease. Curr Cardiovasc Imaging Rep 2017;10:15.

9 Dreisbach JG, Nicol ED, Roobottom CA, et al. Challenges in delivering computed tomography coronary angiography as the firstline test for stable chest pain. Heart 2018;104:921-7.

10 Demaerschalk BM, Bobrow BJ, Raman R, et al. Ct interpretation in a telestroke network: agreement among a spoke radiologist, hub vascular neurologist, and hub neuroradiologist. Stroke 2012;43:3095-7.

11 Mafalanka F, Etard C, Rehel JL, et al. Establishment of diagnostic reference levels in cardiac CT in France: a need for patient dose optimisation. Radiat Prot Dosimetry 2015;164:116-9.

12 Castellano IA, Nicol ED, Bull RK, et al. A prospective national survey of coronary CT angiography radiation doses in the United Kingdom. $J$ Cardiovasc Comput Tomogr 2017;11:268-73.

13 Stocker TJ, Deseive S, Leipsic J, et al. Reduction in radiaTion exposure iN cardiovascular computed tomography imaging: results from the prospective multicenter registry on radiaTion dose estimates of cardiac CT anglOgraphy iN daily practice iN 2017 (PROTECTION VI). Eur Heart J 2018;39:3715-23.

14 Stocker TJ, Leipsic J, Hadamitzky M, et al. Application of Low Tube Potentials in CCTA: Results from PROTECTION VI Study. JACC Cardiovasc Imaging 2020;13:425-434.

15 Suchá D, Tuncay V, Prakken NHJ, et al. Does the aortic annulus undergo conformational change throughout the cardiac cycle? A systematic review. Eur Heart J Cardiovasc Imaging 2015;16:jev210-1317.

16 Jurencak T, Turek J, Kietselaer BLJH, et al. MDCT evaluation of aortic root and aortic valve prior to TAVI. What is the optimal imaging time point in the cardiac cycle? Eur Radiol 2015;25:1975-83.

17 Baumgartner H, Falk V, Bax JJ, et al. 2017 ESC/EACTS guidelines for the management of valvular heart disease. Eur Heart $J$ 2017;38:2739-91.

18 Francone M, Budde RPJ, Bremerich J, et al. CT and MR imaging prior to transcatheter aortic valve implantation: standardisation of scanning protocols, measurements and reporting - a consensus document by the European Society of cardiovascular radiology (ESCR). Eur Radiol 2019;2.

19 Rossi A, De Cecco CN, Kennon SRO, et al. CT angiography to evaluate coronary artery disease and revascularization requirement before trans-catheter aortic valve replacement. J Cardiovasc Comput Tomogr 2017;11:338-46.

20 Annoni AD, Andreini D, Pontone G, et al. CT angiography prior to TAVI procedure using third-generation scanner with wide volume coverage: feasibility, renal safety and diagnostic accuracy for coronary tree. Br J Radiol 2018;91:20180196.

21 Hansson NH, Sörensen J, Harms HJ, et al. Metoprolol reduces hemodynamic and metabolic overload in asymptomatic aortic valve stenosis patients: a randomized trial. Circ Cardiovasc Imaging 2017;10:e006557.

22 Rossi A, Temporelli PL, Cicoira M, et al. Beta-blockers can improve survival in medically-treated patients with severe symptomatic aortic stenosis. Int J Cardiol 2015;190:15-17. 